\title{
Gas density structure of supersonic flows impinged on by thin blades for laser- plasma accelerator targets
}

Cite as: Phys. Fluids 32, 066108 (2020); https://doi.org/10.1063/5.0005888

Submitted: 25 February 2020 • Accepted: 05 June 2020 • Published Online: 30 June 2020

(D) L. Fan-Chiang, (iD H.-S. Mao, (iD) H.-E. Tsai, et al.

CrossMark

\section{ARTICLES YOU MAY BE INTERESTED IN}

Control of quasi-monoenergetic electron beams from laser-plasma accelerators with adjustable shock density profile

Physics of Plasmas 25, 043107 (2018); https://doi.org/10.1063/1.5023694

A compact, high resolution energy, and emittance diagnostic for electron beams using active plasma lenses

Applied Physics Letters 116, 234108 (2020); https://doi.org/10.1063/5.0005114

Perspectives on the generation of electron beams from plasma-based accelerators and their near and long term applications

Physics of Plasmas 27, 070602 (2020); https://doi.org/10.1063/5.0004039

Alp Author Services

English Language Editing

High-quality assistance from subject specialists

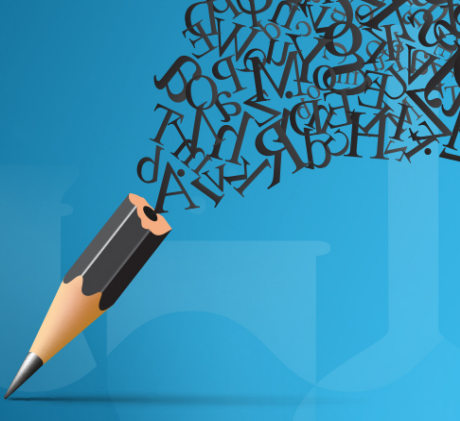




\title{
Gas density structure of supersonic flows impinged on by thin blades for laser-plasma accelerator targets
}

\author{
Cite as: Phys. Fluids 32, 066108 (2020); doi: 10.1063/5.0005888 \\ Submitted: 25 February 2020 - Accepted: 5 June 2020 • \\ Published Online: 30 June 2020

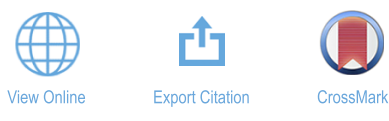

L. Fan-Chiang, ${ }^{1,2, a)}$ (D) H.-S. Mao, (D) H.-E. Tsai, ' (D) T. Ostermayr, (D) K. K. Swanson, ${ }^{1,2}$ (D) S. K. Barber,

S. Steinke,' (D) J. van Tilborg, ' (D) C. G. R. Geddes, ' (D) and W. P. Leemans ${ }^{1, b)}$ (D)

\author{
AFFILIATIONS \\ ${ }^{1}$ Lawrence Berkeley National Laboratory, Berkeley, California 94720, USA \\ ${ }^{2}$ University of California, Berkeley, Berkeley, California 94720, USA
}

\begin{abstract}
a) Author to whom correspondence should be addressed: Ifanchiang@|bl.gov
${ }^{b)}$ Now at: Deutsches Elektronen-Synchrotron (DESY), Hamburg, Germany.
\end{abstract}

\begin{abstract}
Density transition injection is an effective technique for controllably loading electrons into a trapped phase for laser plasma accelerators. One common technique to achieve the required fluid structure is to impinge a thin blade on the plume of a supersonic nozzle. Density transitions induced in this way are often assumed to be bow shocks and therefore sharp, but simulations and fluorescence measurements presented in this work show that in many cases of interest, the density transition accessible to a laser propagating transverse to the shock is an intercepting shock, and therefore, shock thickness and density vary with pressure, laser height, and blade position. The fluid dynamics of a supersonic nozzle impinged on by a thin, flat object are explored through simulations and relevant features are verified via planar laser-induced fluorescence measurements. The implications of the results for tuning electron beam injectors in laser plasma accelerators are discussed.
\end{abstract}

Published under license by AIP Publishing. https://doi.org/10.1063/5.0005888

\section{INTRODUCTION}

As laser plasma accelerator (LPA) technology ${ }^{1-9}$ becomes more mature, controlled injection mechanisms to produce quality electron beams become increasingly important for a variety of applications, such as free electron lasers, ${ }^{10-13}$ Thomson photon sources, ${ }^{14-23}$ and the first injection stage for advanced particle colliders. ${ }^{24,25}$ One popular injection mechanism involves using a thin blade impinging on a supersonic nozzle. ${ }^{16,20,26-47}$ This method creates fluid structures in the gas flow that allow for the controlled injection of electrons into an accelerating structure, either through a density down-ramp, where the phase velocity of the wake is temporarily reduced to allow the electrons to become trapped, ${ }^{26,29,32}$ or a sharp density transition, where the electrons are injected into an accelerating phase through the sudden wake volume change. ${ }^{28}$

The fluid structure by which electrons are injected can have implications on beam quality, such as electron charge and energy spread. The density transition length, $\delta$, compared to the plasma wavelength, $\lambda_{p}$, where $\delta=\Delta n_{e} /\left(\nabla n_{e}\right)_{\max }$ and $\lambda_{p}(\mu \mathrm{m})$ $=3.3 \times 10^{10} \sqrt{n_{e}\left(\mathrm{~cm}^{-3}\right)}$ determine beam properties, most notably the energy spread. Here, $\Delta n_{e}$ is the difference between maximum and minimum densities across the transition, and $\left(\nabla n_{e}\right)_{\max }$ is the maximum gradient. The density down-ramp regime is defined by $\lambda_{p} \ll \delta$, while the sharp density transition regime is defined by $\lambda_{p}>\delta$.

Although commonly used, the performance of electron beams injected from blade-induced density transitions has varied widely due to uncharacterized sources of variation. In an effort to optimize tunability, various studies have characterized the density transition as a function of the blade position relative to the nozzle exit. These studies found that the shock angle varies dramatically with the blade position, which can result in beam steering. ${ }^{34,38,43}$ Studies correcting for the angle by rotating the gas jet did not eliminate the dependence of beam quality on the blade position. ${ }^{43}$ Since both down-ramp injection and shock injection theories predict that the 
effectiveness of electron injection is highly dependent on density transition length, several groups have attempted to measure this length, especially at blade positions at which optimal beam conditions are achieved. These measurements have varied widely, ranging from $2.5 \mu \mathrm{m}$ to over $100 \mu \mathrm{m} .{ }^{31,32,39,43}$ The use of different definitions of shock length further hinders comparison.

In addition to studying the shock transition length, attempts have been made to characterize the underlying physics of the flow. The study of the mechanics of the shock production has been hindered by the difficulty of measuring the gas density profile with traditional methods. The plasma community routinely uses interferometry and shadowgraphy to measure gas profiles. ${ }^{20,34,35,39,44,48,49}$ These techniques, however, are contrast-limited in the low neutral density $\left(n \sim 10^{18} \mathrm{~cm}^{-3}\right)$ regimes required to operate LPAs. Often, in order to increase signal-to-noise ratios, an intense laser is used to ionize the gas to form a plasma, which has a higher refractive index than neutral gas. This technique, however, measures only the ionized portion, often tens of micrometers thin, leaving neutral density, and therefore the rest of the plume, in the dark.

Another challenge posed by blade-impinged gas plumes is that they are asymmetric. Interferometric measurements and shadowgraphy alike are line-integrated measurements that project phase variation along the propagation direction of the probe beam and can blur small features. Additionally, conversion to density requires an Abel inversion that assumes cylindrical symmetry, a symmetry that blade-impinged plumes do not have. In order to capture asymmetric gas plumes, tomographic measurements have been made. ${ }^{20,36,46}$ Applying tomography has its own challenges. In addition to also requiring either higher neutral density or ionization for good contrast, tomography requires images taken from various angles, a degree of freedom not available to many experimental configurations. These images must then be stitched together in post-processing, making the method cumbersome as an online diagnostic.

Simulations regarding shock-assisted injection have overwhelmingly been particle-in-cell simulations of the narrow region in which the laser interacts with the gas density transition. ${ }^{39-42,47}$ The results can conveniently be compared to interferograms of the same field of view. ${ }^{27,37}$ These simulations, like the interferogram images, principally capture the plasma, leaving neutral density and the rest of the plume out. Detailed flow simulations have been performed for conical gas targets, ${ }^{50-52}$ however, neutral flow studies showing the dynamics of the gas plume as a function of blade position have not, until now, been published.

For this paper, we aimed to image and characterize the formation of the shocks measured in various laser plasma experiments. We report results of planar laser-induced fluorescence (PLIF) ${ }^{53,54}$ measurements and flow simulations of the entire plume produced from flows impinged by blades with varying positions. To our knowledge, this is the first time PLIF has been applied to the study of laser plasma accelerator gas jet targets. PLIF fluoresces large twodimensional (2D) sheets of the gas, and thus reveals the plume structure of the full plume in a single shot with minimal post-processing. The technique surmounts many of the challenges posed by traditional gas density profile characterization methods. Since the images are effectively cross sections of the flow, resolution is only imagingsystem-limited and does not assume symmetry. Three-dimensional
(3D) images can be gathered easily. The corresponding fluid simulations presented in this paper show many changes in macroscopic flow characteristics with the blade position, while analysis by the method of characteristics shows how multiple types of shocks are formed.

\section{JET WITH THE BLADE PLUME STRUCTURE}

\section{A. Previous evidence of intercepting shocks in LPA gas jet targets}

The density transition generated by an impinging blade is often assumed to be sharp $\left(\lambda_{p}>\delta\right)$ and due to a bow shock at the blade, like that shown in Fig. 1(a). In the presence of a bow shock, Mott-Smith shock thickness theory, which provides estimates for shock thickness using the Boltzmann equation, can be used to compare $\delta$ to $\lambda_{p}$ and it generally confirms a sharp density transition. ${ }^{55}$ Experimental evidence, ${ }^{38,43}$ however, has suggested that for more-than-glancing interception of the blade with the gas plume, the mechanism for generating the accessible density drop is not a bow shock.

Evidence of the formation of intercepting shocks in LPA targets was first identified and published by Lawrence Berkeley National Laboratory's BELLA Center. ${ }^{38,43}$ In these studies, a conical nozzle with a design Mach number of $\sim 2.5$, was impinged on by a blade with an adjustable position, as shown in Fig. 2. The range of motion

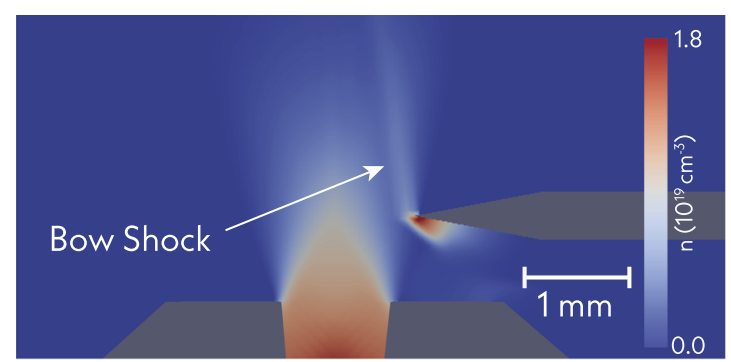

(a)Bow shock

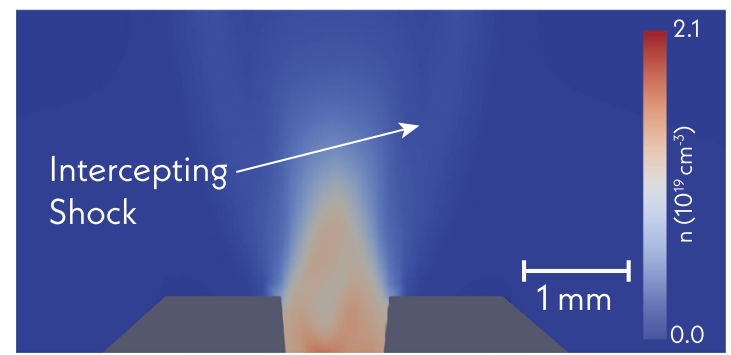

(b)Intercepting shock

FIG. 1. (a) Simulation showing the bow shock that is accessible to an ionizing laser beam that is formed when the blade grazes the gas plume and does not cover over the nozzle exit. Here, $-20 \%$ blade coverage is shown, where $0 \%$ is taken to be the position of the blade when its edge is just above the edge of the mouth of the nozzle. (b) shows a simulation of an intercepting shock formed from a pressure boundary between the plume and local ambient pressure. Density color scales have been saturated to highlight relevant features. 


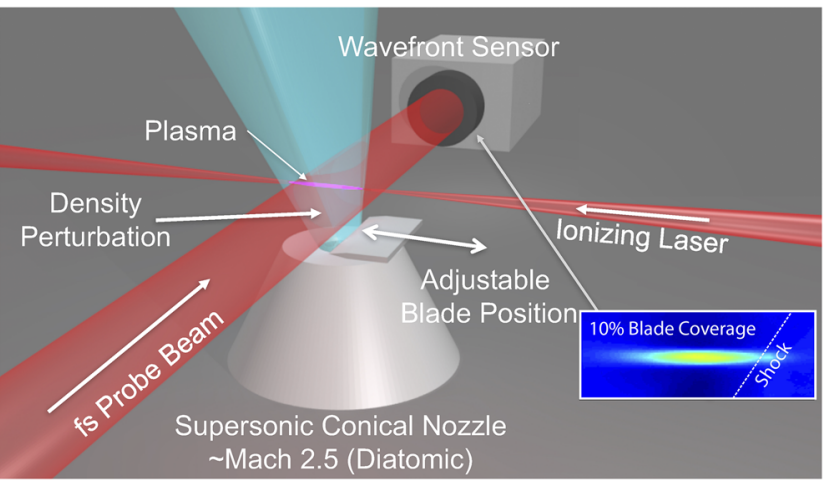

FIG. 2. Schematic of the experimental setup that consisted of a Mach 2.5 conical nozzle obstructed by an orthogonal blade with an adjustable position. In previous experiments, ${ }^{38,43}$ a fs transverse probe beam intersected the laser/plasma interaction onto a wavefront sensor to obtain density information. The inset wavefront image shows a density perturbation tilting toward the blade as a result of $10 \%$ blade coverage. In the present study, PLIF measurements use a fluorescing laser co-linear with the ionizing laser depicted here and an electron-multiplying charge-coupled device in place of the wavefront sensor.

of the blade allowed it to be moved from a position in which the blade did not cover over the nozzle exit but grazed the expanding gas plume ( $-20 \%$ blade coverage), to a position in which the blade completely covered over the nozzle exit ( $100 \%$ blade coverage). $0 \%$ blade coverage corresponds to the edge of the blade directly above the laser-upstream edge of the nozzle exit. An ionizing laser propagated from the blade side of the nozzle. Transverse to the ionizing laser, a femtosecond (fs) probe sampled the plasma and was imaged onto a wavefront sensor. This sensor provided a phase image from which density information could be extracted through a phase retrieval algorithm. ${ }^{56}$ Phase images of the gas plume and the resulting plasma were taken as a function of blade coverage.

Data revealed a localized high density region emanating from the blade edge of the neutral plume. The angle of this feature had strong dependence on blade coverage, as shown in Fig. 3. In contrast to the behavior expected from a bow shock, this feature is shown to sweep past vertical as can be seen in the "10\% Blade Coverage" case. Had this feature been a bow shock, a component of the flow must be directed upstream, which is unphysical. That this feature is not a bow shock is corroborated by a density transition length, $\delta \approx 5 \lambda_{p}$, that is greater than the neutral mean free path. ${ }^{38}$ Additionally, a traditional bow shock would have had a standoff distance from the blade that would decrease with increasing Mach number. Instead, this feature was observed to be attached to the blade edge independent of Mach number. It was hypothesized that the feature encountered by the laser was, instead of a bow shock, an intercepting shock (also called as coalescing shock) arising from re-expansion of the subsonic region behind the bow shock, resulting in coalescence of compression waves. The intercepting shock, or boundary between a plume and a mismatched local ambient pressure, $P_{a m b}$, as shown in Fig. 1(b), is a well-studied phenomenon that occurs when supersonic flows are pressure mismatched with $P_{a m b} .{ }^{57}$ The presence of compression waves before the coalescence into a shock

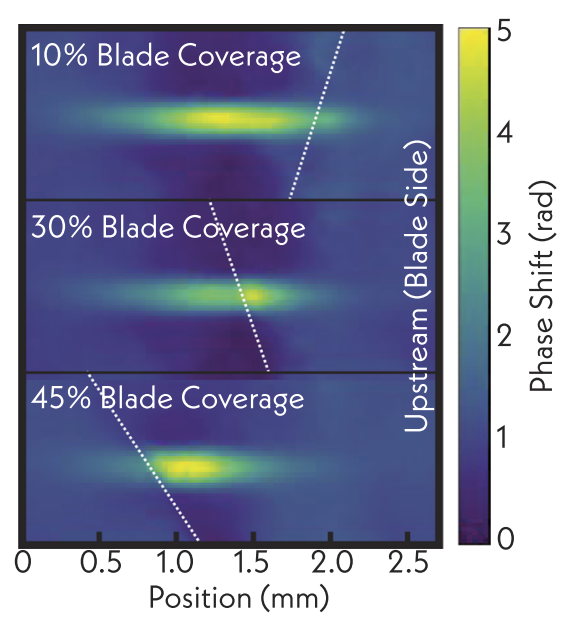

FIG. 3. Wavefront sensor data from previous experiments ${ }^{43}$ showed that the angle of the density perturbation changed for various blade coverage percentages. Note that in the $10 \%$ case, the shock angle tends toward the blade side of the flow, unphysical for bow shocks.

would mean that the density transition length scale experienced by a traversing laser pulse can be large $\left(\delta>\lambda_{p}\right)$ and can change depending on the sampling location as well as the blade position. If this hypothesis were correct, then global flow parameters must be reassessed for LPA injection calculations. More thorough simulations and higher resolution diagnostics were required to confirm this hypothesis.

\section{B. Effects of blade coverage on the fluid structure}

In the current study, gas jet target conditions from previous studies ${ }^{38,43}$ were replicated and examined through simulation, experimentally via PLIF, and analytically by the method of characteristics. Two and three dimensional finite volume simulations of a flow through a conical nozzle with a design Mach number of $\sim 2.5$, impinged on by a blade with adjustable coverage, were conducted using the OpenFOAM compressible Navier-Stokes flow solver, rhoCentralFoam. ${ }^{58}$ Technical details about the computational domain and boundary conditions can be found in the supplementary material. The flow is symmetric about the plane transverse to the blade and it was found that $2 \mathrm{D}$ simulations captured the salient features. In order to model the flow that is expanding from a reservoir through the nozzle into medium vacuum, and therefore is choked, sonic conditions were specified at the nozzle throat with a stagnation pressure of 25 psi-100 psi. The back pressure was varied and the outbound flow was prescribed as homogeneous Neumann boundary conditions. Simulations were performed using the laminar flow approximation and flow is modeled as an ideal gas. Although the flow, with Reynolds number on the order of $1 \times 10^{5}$, is turbulent, the laminar approximation showed agreement with the computationally more expensive simulations that included turbulence to within a plasma wavelength and below experimental resolution. Hence, the simulations presented in this paper were performed using the laminar flow approximation. Heat conduction 


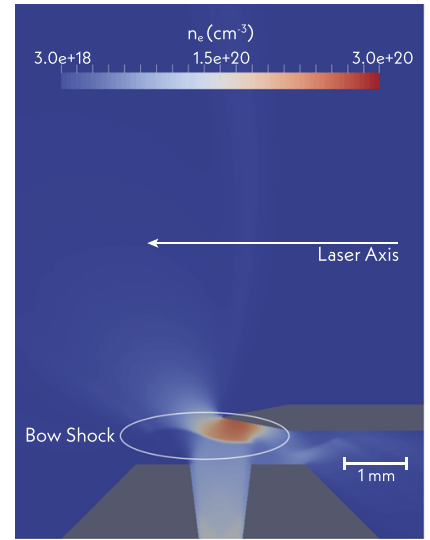

(a)Bow Shock

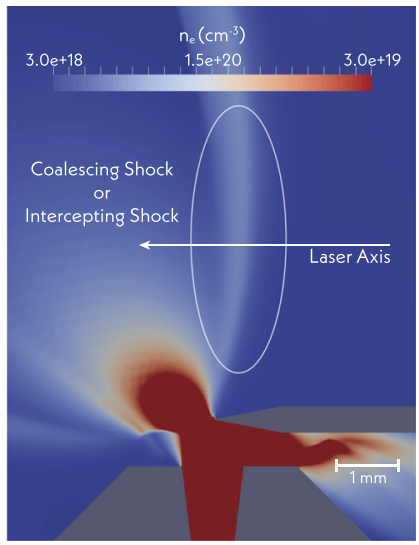

(b)Intercepting Shock
FIG. 4. (a) and (b) both show compressible flow simulations at $50 \%$ blade coverage but with different color scales. (a) shows the flow with a normalized color scale. Here, the initial bow shock separation can be seen in front of the blade. (b) uses a saturated color scale to show the intercepting shock from the re-expansion of the subsonic region behind the bow shock.

and radiation effects are not significant. To study the character of the density perturbation, blade coverages from $-20 \%$ to $120 \%$ were considered.

The simulation for $50 \%$ blade coverage is shown in Fig. 4. Neutral density was converted to plasma density, $n_{e}\left(\mathrm{~cm}^{-3}\right)$ by assuming that the laser pulse fully ionizes the region of flow sampled. The laser pulse originates from the right-hand side above the blade as shown in Fig. 2. The density of the jet is shown in Fig. 4(a) in which the separated bow shock can be seen below the blade. As previously stated, the bow shock was directly accessible only in the case where the blade is adjacent to, but not covering, the nozzle as shown in Fig. 1(a). When the blade coverage was $>0 \%$ the main features of this shock did not travel sufficiently far enough into the flow to be accessed by the traversing laser.
When the blade coverage was $>0 \%$, the density transition that is accessible to the traversing laser was shown to be an intercepting shock. The intercepting shock emerges from the combination of the bow shock and the blade, which together form the throat of a dynamically created virtual nozzle. High pressure gas behind the normal portion of the bow shock, subsonic by definition, reaches a sonic line at the blade, and is subsequently re-accelerated and re-expanded through this virtual nozzle. The re-expansion behind the bow shock closely mimics a sonic nozzle and acts as an underexpanded jet creating an intercepting shock in the plume of the virtual nozzle, as shown in Fig. 4(b).

The intercepting shock forms from coalescing Mach waves that are generated when the pressure from the nozzle is mismatched with the local ambient pressure. Note that the local ambient pressure differs from the back pressure in the chamber that is at high vacuum. This intercepting shock is the experimentally observed density perturbation shown in Fig. 3 and adequately explains the observed reverse perturbation angle.

In order to experimentally explore the $2 \mathrm{D}$ simulated flow features of interest, 2D slices of the gas plume were imaged via PLIF. ${ }^{59} \mathrm{~A}$ schematic of the experimental configuration is shown in Fig. 5. Previous density measurements used a wavefront sensor to image phase distortions through the plasma created by an ionizing laser. ${ }^{38,43}$ As shown in Fig. 3, these measurements gave 1D density line-outs of the plume. The investigation of the structure of the plume would have required many measurements at different heights. More importantly, the resolution required to define the shock length was difficult to obtain using available wavefront sensors. Although interferometry could obtain the sub- $\lambda_{p}$ resolution, the largest obstacle for imaging was target asymmetry. Wavefront measurements assume cylindrical symmetry in order to convert phase differences to density. Such a conversion blurs the features under investigation. In order to properly account for asymmetry, tomography can be performed, however, the resolution of a tomographic reconstruction is determined by the number of angles at which images are obtained, a degree of freedom often not available to many experimental setups. In order to surmount the challenges of these techniques, PLIF, which uses a planar beam to fluoresce a $2 \mathrm{D}$ slice of the plume, was used.

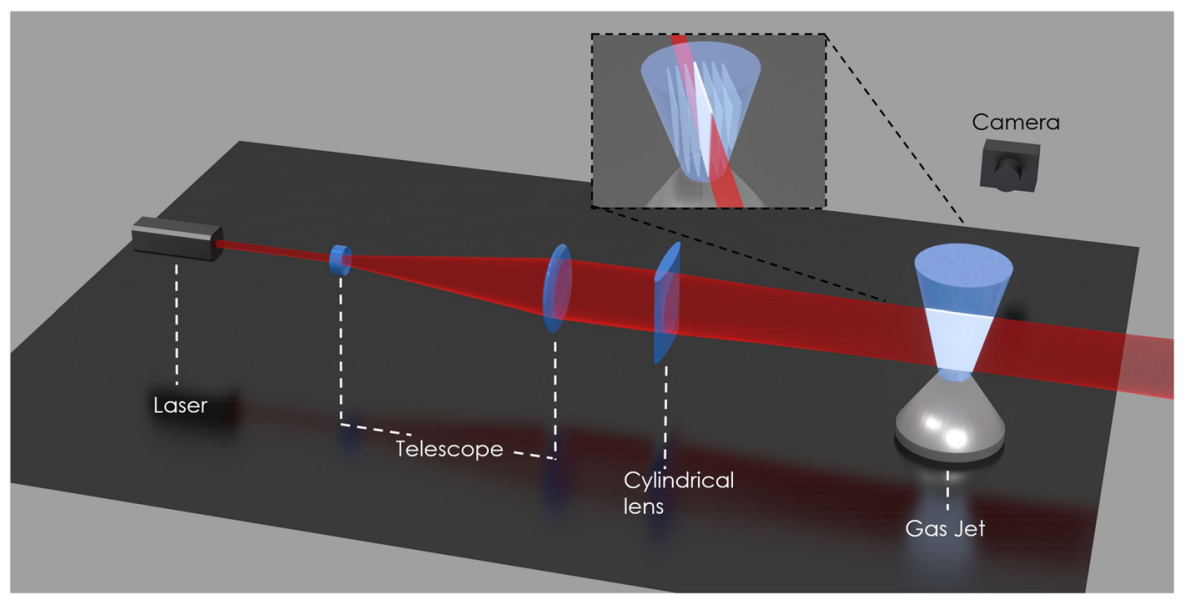

FIG. 5. Schematic of planar laserinduced fluorescence experimental configuration. 


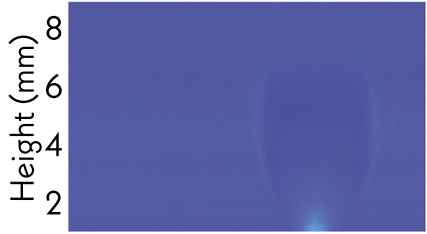

(a) Uncovered

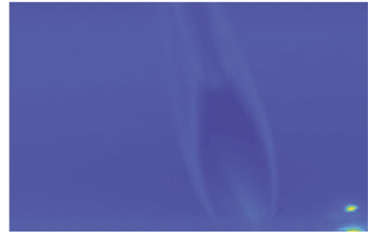

(b) $9 \%$ covered

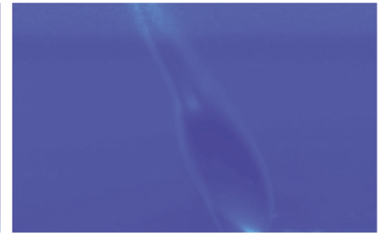

(c) $49 \%$ covered

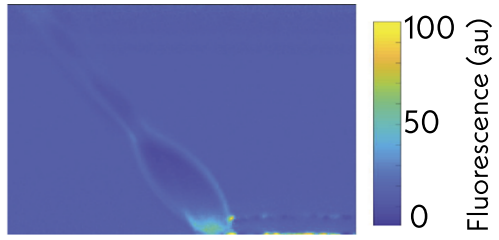

(d) $71 \%$ covered

FIG. 6. PLIF measurements of nozzle with blade configuration. (a) shows the plume with no blade impinging, while in (b) the blade hovers over $9 \%$, (c) over $49 \%$, and (d) over $71 \%$ of the nozzle exit. The image resolution shown is $44 \mu \mathrm{m} / \mathrm{px}$. Insertion of the blade at various positions changes the periodicity of shock diamonds. This indicates a change in the plume internal pressure/density similar to that shown in Fig. 10.

The resolution, $44 \mu \mathrm{m} /$ pixel, was determined by the imaging system, consisting of an electron-multiplying charge-coupled device and a $50 \mathrm{~mm}$ TV lens. The thickness of the fluoresced slice was determined by the width of the focus of the fluorescing beam. Raw images revealed much of the fluid structure, and could be quickly converted to density by calibration to the fluorescence signals of known densities.

In these experiments, argon and trace amounts of acetone were flowed into low vacuum through a nozzle with design Mach number 2.5 and a $1 \mathrm{~mm}$ exit diameter. A blade with an adjustable position was suspended $600 \mu \mathrm{m}$ above the nozzle. A $266 \mathrm{~nm}$ wavelength laser was focused to a $60 \mu \mathrm{m}$ wide, $5 \mathrm{~mm}$ tall line-focus with a $1 \mathrm{~cm}$ Rayleigh length and served as the fluorescing laser sheet.

The resolution enabled by PLIF imaging revealed that the density transition length $\delta$ was on the order of $4 \lambda_{p}$, as shown in a fluorescence intensity line-out of the plume (shown in Fig. 6a) plotted in Fig. 7 , in contrast to the sub- $\lambda_{p}$ transition predicted by MottSmith for a bow shock. In addition, simulations paired with experiments revealed changes in core flow parameters, characteristic of a redefinition of the sonic line and the corresponding intercepting shock.

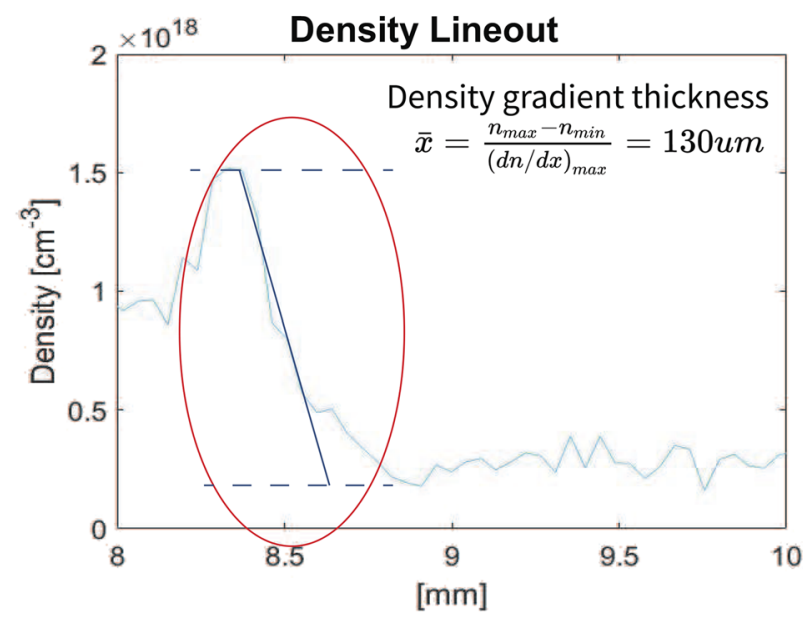

FIG. 7. Lineout of a PLIF measured density gradient. At this density (Ar at $n=2.0 \cdot 10^{17} \mathrm{~cm}^{-3}$ ), $\lambda_{p}$ would be $32 \mu \mathrm{m}$, while the measured density gradient thickness $\delta=130 \mu \mathrm{m}$.
Moreover, simulations paired with experiments revealed the sensitivity of core flow parameters to the blade position and pressure. As shown in Fig. 8, simulations showed that the suspended blade redefined the sonic line of the plume, generating a sonic nozzle external to the original nozzle. Varying the blade position altered this sonic line and redirected the vector of the virtual sonic nozzle. Furthermore, as can be seen by comparing Figs. 8(a) and 8(b), more of the flow went through the bow shock at a normal angle for larger blade coverages. Larger blade coverages effectively reduced the stagnation pressure in the subsonic region and reduced the Mach number needed to match the pressure of the jet plume after re-expansion. Figures 9(a) and 9(b) show that the same effect was generated by changing the input plume pressure while keeping the blade position fixed. Note that the simulations shown in Fig. 9 are 3D, while others shown herein are $2 \mathrm{D}$, and are included here to show contributions to the features discussed, such as the plume angle and density transition length, from 3D effects. Dimensionality did not change the results significantly, as expected since the blade is long compared to the plume scales and analytically the flow is symmetric about the plane

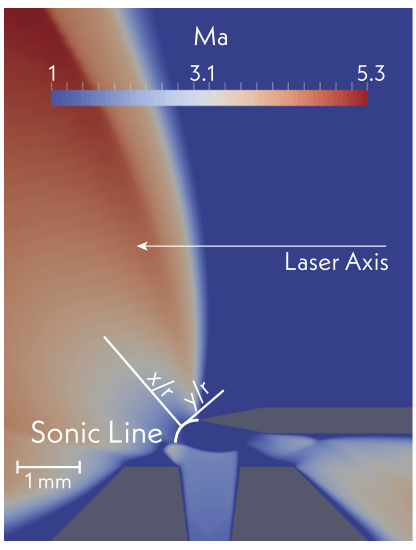

(a) $70 \%$ Blade Coverage

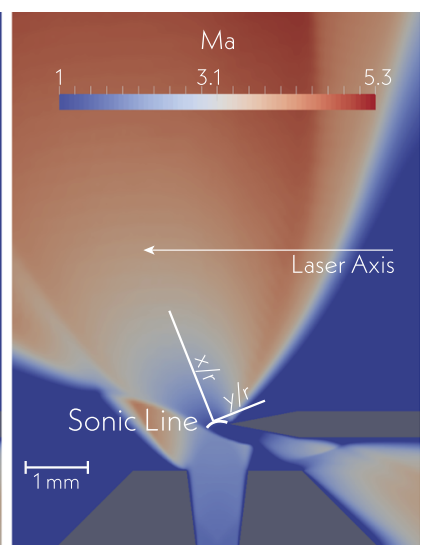

(b) $30 \%$ Blade Coverage
FIG. 8. Simulations showing Mach number at (a) $70 \%$ and (b) $30 \%$ blade coverage. The blade coverage strongly affects the vector of the re-expanded plume along with the fluid structure of the intercepting shock used for electron injection. Note the contour and position of the sonic line between the blade edge and the bow shock. The coordinate system shows the relationship with the method of characteristic simulations shown in Figs. 11 and 12. 


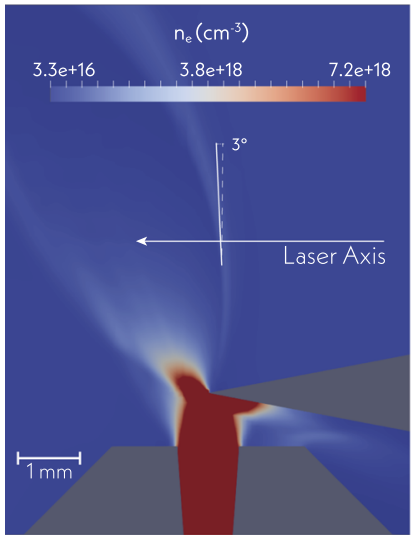

(a) $P_{\text {exit }} / P_{a m b}=15$

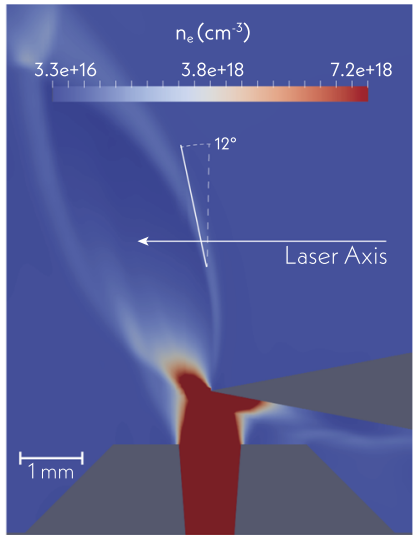

(b) $P_{\text {exit }} / P_{a m b}=11$
FIG. 9. 3D Simulation showing the density of gas flows at varying pressure ratios $P_{\text {exit }} / P_{\text {amb }}$ for a fixed blade position. For a set blade coverage, the angle of the intercepting shock increases with respect to the plume axis as the pressure ratio increases, which is consistent with the method of characteristic results shown in Fig. 13. More details about comparison between 3D simulations and experimental measurements can be found in the supplementary material.

transverse to the blade. Further detail can be found in the supple mentary material. These features were corroborated experimentally via PLIF.

Figure 6 shows PLIF measurements for increasing blade coverages with a fixed reservoir and back pressure. As the blade covered more of the nozzle exit, the sonic line, and therefore plume axis, was visibly tilted. Since measured back (chamber) pressure, $P_{\text {back }}$, and local ambient (near plume) pressure, $P_{a m b}$, differ, calibration simulations were used to correlate the plume shape with pressure ratios $P_{\text {exit }} / P_{a m b}$ and $P_{a m b} / P_{b a c k}$. Variation in shock diamond periodicity indicated a change in $P_{\text {exit }} / P_{a m b}$ for a fixed design Mach number nozzle. For comparison, Fig. 10 shows simulations of the same effect in plumes with no blade and fixed bottle pressure but

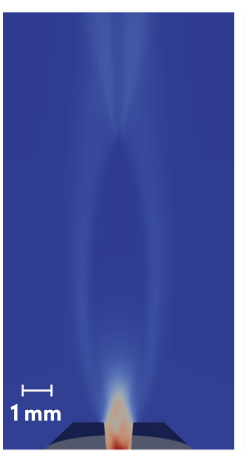

(a)

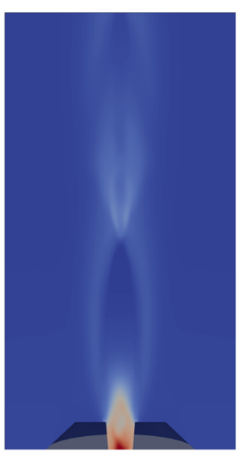

(b)

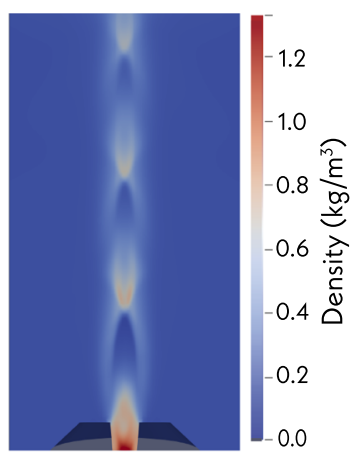

(c)
FIG. 10. Simulation of pressure-mismatched nozzles at different pressure ratios (a) $P_{\text {exit }} / P_{\text {amb }}=4.3$, (b) $P_{\text {exit }} / P_{\text {amb }}=3.3$, and (c) $P_{\text {exit }} / P_{\text {amb }}=2.6$. Note the increase of Mach disk periodicity as $P_{\text {exit }} / P_{\text {amb }}$ decreases. The density in the plume increases with increasing diamond frequency. increasing $P_{a m b}$. Since both bottle and back pressure were held constant in the simulations of blade obstructed plumes and in PLIF experiments, it is clear that characteristic flow parameters, such as stagnant pressure, must be changing with blade positions, resulting in a redefined core flow as though through nozzles of varying design Mach numbers.

Simulation and measurements showed also that the shock tilt encountered by an ionizing laser is determined by a combination of plume axis orientation as a function of the blade position, and pressure ratio $\left(P_{\text {exit }} / P_{a m b}\right)$ tuned by bottle pressure, as shown in Figs. 6, 8, and 9. These two factors combined result in a range of configurations in which the shock tilts above the blade, as had been observed in previous wavefront measurements (Fig. 3). The relationship between shock angle and blade position dependent flow characteristics became clearer when the flow was analyzed by the method of characteristics.

\section{Intercepting shock from coalescing Mach waves by the method of characteristics}

The dynamics of the intercepting shock can be better understood through the analysis of the flow by the method of characteristics, which predicts the flow field by tracing out the interaction of upstream supersonic regions via their characteristics or Mach lines. The intercepting shock is formed from Mach waves coalescing into a shock wave. Upstream of the coalescing point, the density transition is relatively smooth $\left(\gg \lambda_{p}\right)$. Beyond the coalescing point, the density transition can be taken to be sharp $\left(\sim \lambda_{p}\right)$. The Mach waves can be traced out from a nozzle using the method of characteristics starting from a sonic line at the throat as shown in Figs. 11 and $12 .{ }^{60,61}$ In contrast to the finite volume method where the spatial resolution is determined by the grid size, the resolution for the method of characteristics is determined by the number of characteristic lines used. Note that all spatial dimensions are normalized to the throat half-width, $r$. An initial expansion fan is generated as the flow accelerates around the corner of the sonic nozzle. The characteristic lines pass through the centerline, effectively a reflection in the symmetrized model, and form an expansion region of flow where

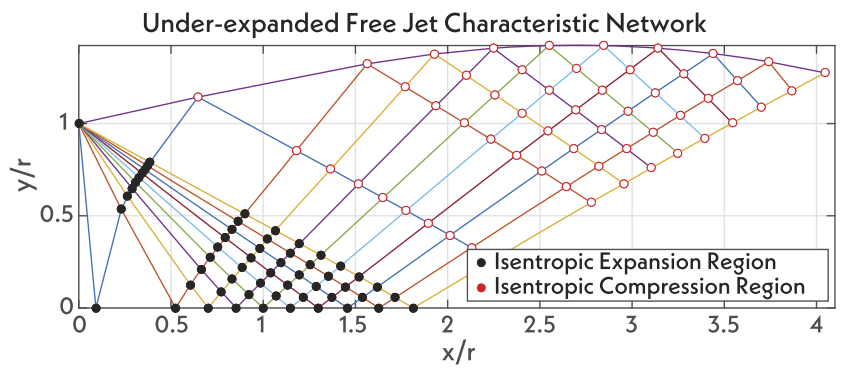

FIG. 11. The characteristic network created by a sonic free jet originates from the corner of the nozzle as the flow begins to expand. This creates an isentropic expansion region, shown with the black solid markers, which first reflects off the centerline $(y / r=0)$. Here, $y / r$ is the transverse direction normalized to the nozzle exit radius, corresponding to the sonic line, as labeled in Fig. 8, and $x / r$ is along the axis of the plume. As the flow is expanded, the characteristic lines are reflected a second time off the free-boundary back toward the centerline. A converging network is formed, which acts as an isentropic compression fan. 


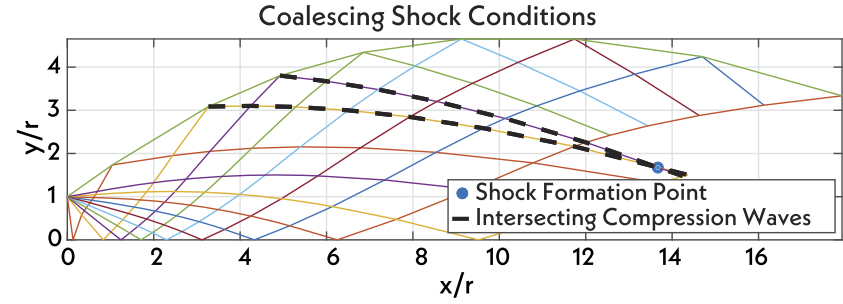

FIG. 12. Intercepting characteristics coalesce into a shock wave at sufficiently high $P_{\text {exit }} / P_{\text {amb }}$. The trajectory of the coalesced shock is determined by its composite compression waves. Following the initial formation shown here, subsequent waves will merge with the shock creating a stronger shock.

they continuously accelerate. As the flow expands, the flow pressure will eventually match the local ambient pressure, at which point a free-boundary is created and the characteristic lines are reflected back toward the center of the flow. These converging characteristic lines create an isentropic compression fan. The gas flow before and after an isentropic compression fan is exactly the same as if the flow had seen a shock wave. There are two primary differences between traveling through a shock and traveling through a compression fan. In contrast to traveling through a shock, traveling through a compression fan results in (1) an increase in the stagnation pressure and (2) a density transition with a length scale that can be much greater than that predicted by Mott-Smith. The re-expansion of the sonic nozzle from the blade can be simplified to a sonic orifice with a flat sonic line expanding into ambient gas. This is known as an under-expanded free-jet and has been extensively studied.

The intersection of the Mach waves in the compression region signals shock formation. As the ratio of exit pressure to local ambient pressure, $P_{\text {exit }} / P_{a m b}$, is increased, the curvature of the free-boundary becomes more severe and the plume expands. The reflected characteristic line's convergence also becomes steeper. At a high enough $P_{\text {exit }} / P_{a m b}$, the characteristic lines will intersect at which point they coalesce and form a shock wave. As the shock wave propagates, more characteristic lines will coalesce with the shock, creating an increasingly stronger shock wave, and thus a sharper drop in the density at the interface. The formation of this shock from two converging characteristics is shown in Fig. 12.

By varying $P_{\text {exit }} / P_{a m b}$, the point at which the compression fan coalesces into a shock wave can be traced out with respect to the sonic line position. Figure 13 shows the shock formation point for several simulations, as shown in Fig. 12, for pressure ratios up to 230. Approximately, 100 characteristic lines were used to resolve the formation location. Shock formation does not occur until the pressure ratio is $\sim 5.5$ for the simple free-jet model. As $P_{\text {exit }} / P_{a m b}$ increases, the plume widens (green line in Fig. 12). As a result, the intersection point of compression waves occurs later in the flow and a distinct shock forms further down (increased $x / r$ ) and further out (increased $y / r$ ) from the sonic line. The formation point and the sonic line grow more rapidly in the transverse direction, $y / r$, manifesting in the full flow field as a wider plume, and thus an angle change of the intercepting shock. Although ratios differ slightly for re-expansion in the blade configuration due to the curved sonic line, as indicated in Fig. 8(a), as well as the asymmetry of the flow not captured in this analysis, this delay explains

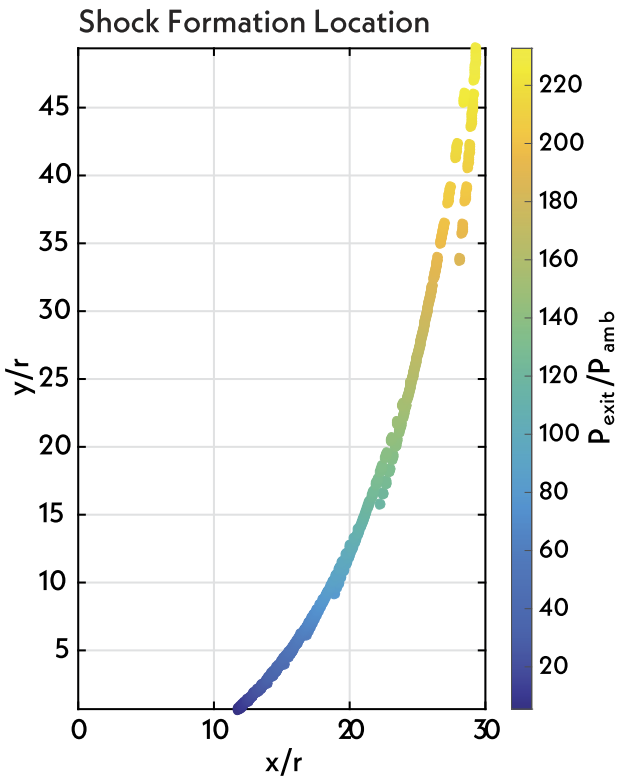

FIG. 13. The shock formation point location as a function of $P_{\text {exit }} / P_{\text {amb }}$. As the pressure ratio increases, the shock formation point travels transverse to the nozzle exit plane. Note that the discontinuities in the curve stem from different characteristics causing the initial intersection in the simulation.

both the displaced shock formation point and the expanding plume manifested in simulations as well as in PLIF measurements described in Sec. II B.

\section{CONSIDERATIONS FOR LPA TARGETS}

The knowledge of how plume characteristics depend on the parameters investigated above may be used in customizing density transitions for LPA targets. The shock angle relative to the ionizing laser has been shown to affect the quality and direction of electron beams. ${ }^{38}$ As discussed above, this angle can be adjusted with the pressure ratio and blade coverage fraction. These parameters, however, affect another important quantity: density. Since blade and bow shock interact to form a secondary virtual nozzle, varying the blade position varies the amount of flow traversing the normal shock upstream of the blade, changing the stagnation pressure of the new subsonic region, and therefore $P_{\text {exit }} / P_{a m b}$. The effect is a change in overall density in the main plume.

Finally, since the density transition accessible to the laser for these parameters is an intercepting shock, and does not coalesce into a shock immediately, it is important to consider the shock formation location. Although not all will be accessible for a fixed laser height, by controlling pressure ratios, the shock formation height can be adjusted. This will alter the shock width and angle, as shown in Fig. 9. The formation of the coalesced intercepting shock strongly depends on $P_{a m b}$, which differs from the global chamber pressure. The measurement of $P_{a m b}$ is difficult in practice. The comparison of measured and simulated gas jets suggests that $P_{a m b}$ is much higher than that measured in the chamber. Further analysis requires simulations that include the domain of the full chamber. 
While 3D simulations are required for exact fluid structures, qualitative rules of thumb can be established: the farther the laser is from the sonic line (i.e., the blade), the shorter the density transition length $(\delta)$ will be since the shock gets stronger with distance. This is at the expense of absolute density $\left(n_{e}\right)$ through natural expansion.

\section{CONCLUSION}

In conclusion, a commonly used supersonic jet-with-blade LPA target, previously assumed to provide a sharp density transition by means of a bow shock, was shown to have a more complex fluid flow than was previously thought, which may affect how these targets are used. 2D and 3D compressible flow simulations, 2D imaging via PLIF, and analysis by the method of characteristics have been conducted to study the density perturbation produced by a blade suspended above a supersonic, conical nozzle. For these conditions, it was revealed that with any appreciable blade coverage, the accessible density perturbation is an intercepting shock. The current study has explained how the density transition length, $\delta$, can potentially be larger than $\lambda_{p}$. As a consequence, comparing $\lambda_{p}$ to Mott-Smith shock thickness theory may not be applicable for predicting the injection mechanism for LPAs under many conditions of interest. More importantly, it has been shown that flow parameters, shock angle, density, and density transition length are highly coupled. Although this coupling may complicate the tuning of jetand-blade LPA targets, the variety of density profiles easily produced by such targets makes them potentially capable of facilitating a wide range of electron beam parameters.

\section{SUPPLEMENTARY MATERIAL}

See the supplementary material for more information about more technical specifications of simulation as well as plots of pressure, temperature, and velocity for the case shown in Fig. 8.

\section{ACKNOWLEDGMENTS}

This work was supported by the Director, Office of Science, Office of High Energy Physics of the U.S. Department of Energy under Contract No. DE-AC02-05CH11231, by the National Science Foundation (NSF) under Contract No. PHY-1415596 and Grant No. DGE 1752814, and by the Department of Energy National Nuclear Security Administration, Defense Nuclear Nonproliferation Grant No. R\&D (NA22).

\section{DATA AVAILABILITY}

Raw data were generated at the Lawrence Berkeley National Laboratory. The data that support the findings of this study are available from the corresponding author upon reasonable request.

\section{REFERENCES}

${ }^{1}$ T. Tajima and J. M. Dawson, “Laser electron accelerator," Phys. Rev. Lett. 43, 267 (1979).

${ }^{2}$ E. Esarey, C. B. Schroeder, and W. P. Leemans, "Physics of laser-driven plasmabased electron accelerators," Rev. Mod. Phys. 81, 1229 (2009).

${ }^{3}$ X. Wang, R. Zgadzaj, N. Fazel, Z. Li, S. A. Yi, X. Zhang, W. Henderson, Y. Y. Chang, R. Korzekwa, H. E. Tsai, C. H. Pai, H. Quevedo, G. Dyer, E. Gaul, M. Martinez, A. C. Bernstein, T. Borger, M. Spinks, M. Donovan, V. Khudik,
G. Shvets, T. Ditmire, and M. C. Downer, "Quasi-monoenergetic laser-plasma acceleration of electrons to $2 \mathrm{GeV}$," Nat. Commun. 4, 1988 (2013).

${ }^{4}$ F. Albert and A. G. R. Thomas, "Applications of laser wakefield accelerator-based light sources,” Plasma Phys. Controlled Fusion 58, 103001 (2016).

${ }^{5}$ H. T. Kim, V. B. Pathak, K. Hong Pae, A. Lifschitz, F. Sylla, J. H. Shin, C. Hojbota, S. K. Lee, J. H. Sung, H. W. Lee, E. Guillaume, C. Thaury, K. Nakajima, J. Vieira, L. O. Silva, V. Malka, and C. H. Nam, "Stable multi-GeV electron accelerator driven by waveform-controlled PW laser pulses," Sci. Rep. 7, 10203 (2017).

${ }^{6}$ J. Wenz, A. Döpp, K. Khrennikov, S. Schindler, M. F. Gilljohann, H. Ding, J. Götzfried, A. Buck, J. Xu, M. Heigoldt, W. Helml, L. Veisz, and S. Karsch, "Dualenergy electron beams from a compact laser-driven accelerator," Nat. Photonics 13, 263 (2019).

${ }^{7}$ A. J. Gonsalves, K. Nakamura, J. Daniels, C. Benedetti, C. Pieronek, T. C. H. De Raadt, S. Steinke, J. H. Bin, S. S. Bulanov, J. Van Tilborg, C. G. R. Geddes, C. B. Schroeder, C. Tóth, E. Esarey, K. Swanson, L. Fan-Chiang, G. Bagdasarov, N. Bobrova, V. Gasilov, G. Korn, P. Sasorov, and W. P. Leemans, "Petawatt laser guiding and electron beam acceleration to $8 \mathrm{GeV}$ in a laser-heated capillary discharge waveguide," Phys. Rev. Lett. 122, 084801 (2019).

${ }^{8}$ J. Faure, D. Gustas, D. Guénot, A. Vernier, F. Böhle, M. Ouillé, S. Haessler, R. Lopez-Martens, and A. Lifschitz, "A review of recent progress on laser-plasma acceleration at kHz repetition rate," Plasma Phys. Controlled Fusion 61, 014012 (2019).

${ }^{9}$ T. Tajima and V. Malka, "Laser plasma accelerators," Plasma Phys. Controlled Fusion 62, 034004 (2020).

${ }^{10}$ Z. Huang, Y. Ding, and C. B. Schroeder, “Compact X-ray free-electron laser from a laser-plasma accelerator using a transverse-gradient undulator,” Phys. Rev. Lett. 109, 204801 (2012).

${ }^{11}$ A. R. Maier, A. Meseck, S. Reiche, C. B. Schroeder, T. Seggebrock, and F. Grüner, "Demonstration scheme for a laser-plasma-driven free-electron laser," Phys. Rev. X 2, 031019 (2012).

${ }^{12}$ J. van Tilborg, S. K. Barber, F. Isono, C. B. Schroeder, E. Esarey, and W. P. Leemans, "Free-electron lasers driven by laser plasma accelerators," AIP Conf. Proc. 1812, 020002 (2017).

${ }^{13}$ I. Gadjev, N. Sudar, M. Babzien, J. Duris, P. Hoang, M. Fedurin, K. Kusche, R. Malone, P. Musumeci, M. Palmer, I. Pogorelsky, M. Polyanskiy, Y. Sakai, C. Swinson, O. Williams, and J. B. Rosenzweig, "An inverse free electron laser acceleration-driven Compton scattering X-ray source,” Sci. Rep. 9, 532 (2019).

${ }^{14}$ K. Ta Phuoc, S. Corde, C. Thaury, V. Malka, A. Tafzi, J. P. Goddet, R. C. Shah, S. Sebban, and A. Rousse, "All-optical Compton gamma-ray source," Nat. Photonics 6, 308 (2012).

${ }^{15}$ S. Chen, N. D. Powers, I. Ghebregziabher, C. M. Maharjan, C. Liu, G. Golovin, S. Banerjee, J. Zhang, N. Cunningham, A. Moorti, S. Clarke, S. Pozzi, and D. P. Umstadter, "MeV-energy x rays from inverse compton scattering with laser-wakefield accelerated electrons,” Phys. Rev. Lett. 110, 155003 (2013).

${ }^{16}$ C. G. Geddes, S. Rykovanov, N. H. Matlis, S. Steinke, J.-L. Vay, E. H. Esarey, B. Ludewigt, K. Nakamura, B. J. Quiter, C. B. Schroeder, C. Toth, and W. P. Leemans, "Compact quasi-monoenergetic photon sources from laser-plasma accelerators for nuclear detection and characterization," Nucl. Instrum. Methods Phys. Res., Sect. B 350, 116 (2015).

${ }^{17}$ H.-E. Tsai, X. Wang, J. M. Shaw, Z. Li, A. V. Arefiev, X. Zhang, R. Zgadzaj, W. Henderson, V. Khudik, G. Shvets, and M. C. Downer, "Compact tunable Compton x-ray source from laser-plasma accelerator and plasma mirror," Phys. Plasmas 22, 023106 (2015).

${ }^{18}$ K. Khrennikov, J. Wenz, A. Buck, J. Xu, M. Heigoldt, L. Veisz, and S. Karsch, "Tunable all-optical quasimonochromatic Thomson X-ray source in the nonlinear regime," Phys. Rev. Lett. 114, 195003 (2015).

${ }^{19}$ J. Luo, M. Chen, M. Zeng, J. Vieira, L. L. Yu, S. M. Weng, L. O. Silva, D. A. Jaroszynski, Z. M. Sheng, and J. Zhang, "A compact tunable polarized X-ray source based on laser-plasma helical undulators," Sci. Rep. 6, 29101 (2016).

${ }^{20}$ G. Golovin, S. Banerjee, S. Chen, N. Powers, C. Liu, W. Yan, J. Zhang, P. Zhang, B. Zhao, and D. Umstadter, "Control and optimization of a staged laser-wakefield accelerator,” Nucl. Instrum. Methods Phys. Res., Sect. A 830, 375 (2016).

${ }^{21}$ C. Yu, R. Qi, W. Wang, J. Liu, W. Li, C. Wang, Z. Zhang, J. Liu, Z. Qin, M. Fang, K. Feng, Y. Wu, Y. Tian, Y. Xu, F. Wu, Y. Leng, X. Weng, J. Wang, F. Wei, Y. Yi, $\mathrm{Z}$. Song, R. Li, and Z. Xu, "Ultrahigh brilliance quasi-monochromatic $\mathrm{MeV} \gamma$-rays 
based on self-synchronized all-optical Compton scattering," Sci. Rep. 6, 29518 (2016).

${ }^{22}$ W. Yan, C. Fruhling, G. Golovin, D. Haden, J. Luo, P. Zhang, B. Zhao, J. Zhang, C. Liu, M. Chen, S. Chen, S. Banerjee, and D. Umstadter, "High-order multiphoton Thomson scattering," Nat. Photonics 11, 514 (2017).

${ }^{23}$ S. Schindler, A. Döpp, H. Ding, M. Gilljohann, J. Götzfried, and S. Karsch, "Tunable X-ray source by Thomson scattering during laser-wakefield acceleration," Proc. SPIE 11037, 22 (2019).

${ }^{24}$ C. B. Schroeder, E. Esarey, C. G. R. Geddes, C. Benedetti, and W. P. Leemans, "Physics considerations for laser-plasma linear colliders," Phys. Rev. Spec. Top.Accel. Beams 13, 101301 (2010).

${ }^{25}$ S. Steinke, J. van Tilborg, C. Benedetti, C. G. R. Geddes, C. B. Schroeder, J. Daniels, K. K. Swanson, A. J. Gonsalves, K. Nakamura, N. H. Matlis, B. H. Shaw, E. Esarey, and W. P. Leemans, "Multistage coupling of independent laser-plasma accelerators," Nature 530, 190 (2016).

${ }^{26}$ S. Bulanov, N. Naumova, F. Pegoraro, and J. Sakai, "Particle injection into the wave acceleration phase due to nonlinear wake wave breaking," Phys. Rev. E 58, R5257 (1998).

${ }^{27}$ S. Semushin and V. Malka, "High density gas jet nozzle design for laser target production,” Rev. Sci. Instrum. 72, 2961 (2001).

${ }^{28}$ H. Suk, H. J. Lee, and I. S. Ko, "Generation of high-energy electrons by a femtosecond terawatt laser propagating through a sharp downward density transition," J. Opt. Soc. Am. B 21, 1391 (2004).

${ }^{29}$ C. G. R. Geddes, K. Nakamura, G. R. Plateau, C. Toth, E. Cormier-Michel, E. Esarey, C. B. Schroeder, J. R. Cary, and W. P. Leemans, "Plasma-densitygradient injection of low absolute-momentum-spread electron bunches," Phys. Rev. Lett. 100, 215004 (2008).

${ }^{30}$ K. Schmid, A. Buck, C. M. S. Sears, J. M. Mikhailova, R. Tautz, D. Herrmann, M. Geissler, F. Krausz, and L. Veisz, "Density-transition based electron injector for laser driven wakefield accelerators," Phys. Rev. Spec. Top.-Accel. Beams 13, 091301 (2010).

${ }^{31}$ J. Faure, C. Rechatin, O. Lundh, L. Ammoura, and V. Malka, "Injection and acceleration of quasimonoenergetic relativistic electron beams using density gradients at the edges of a plasma channel," Phys. Plasmas 17, 083107 (2010).

${ }^{32}$ A. J. Gonsalves, K. Nakamura, C. Lin, D. Panasenko, S. Shiraishi, T. Sokollik, C. Benedetti, C. B. Schroeder, C. G. R. Geddes, J. van Tilborg, J. Osterhoff, E. Esarey, C. Toth, and W. P. Leemans, "Tunable laser plasma accelerator based on longitudinal density tailoring," Nat. Phys. 7, 862 (2011).

${ }^{33}$ A. Buck, J. Wenz, J. Xu, K. Khrennikov, K. Schmid, M. Heigoldt, J. M. Mikhailova, M. Geissler, B. Shen, F. Krausz, S. Karsch, and L. Veisz, "Shock-front injector for high-quality laser-plasma acceleration," Phys. Rev. Lett. 110, 185006 (2013).

${ }^{34}$ C. Thaury, E. Guillaume, A. Lifschitz, K. Ta Phuoc, M. Hansson, G. Grittani, J. Gautier, J.-P. Goddet, A. Tafzi, O. Lundh, and V. Malka, "Shock assisted ionization injection in laser-plasma accelerators," Sci. Rep. 5, 16310 (2015).

${ }^{35}$ E. Guillaume, A. Döpp, C. Thaury, K. Ta Phuoc, A. Lifschitz, G. Grittani, J. P. Goddet, A. Tafzi, S. W. Chou, L. Veisz, and V. Malka, "Electron rephasing in a laser-wakefield accelerator," Phys. Rev. Lett. 115, 155002 (2015).

${ }^{36}$ J. P. Couperus, A. Köhler, T. A. Wolterink, A. Jochmann, O. Zarini, H. M. Bastiaens, K. J. Boller, A. Irman, and U. Schramm, "Tomographic characterisation of gas-jet targets for laser wakefield acceleration," Nucl. Instrum. Methods Phys. Res., Sect. A 830, 504 (2016).

${ }^{37}$ O. Kononenko, N. C. Lopes, J. M. Cole, C. Kamperidis, S. P. Mangles, Z. Najmudin, J. Osterhoff, K. Poder, D. Rusby, D. R. Symes, J. Warwick, J. C. Wood, and C. A. Palmer, "2D hydrodynamic simulations of a variable length gas target for density down-ramp injection of electrons into a laser wakefield accelerator," Nucl. Instrum. Methods Phys. Res., Sect. A 829, 125 (2016).

${ }^{38}$ K. K. Swanson, H. E. Tsai, S. K. Barber, R. Lehe, H. S. Mao, S. Steinke, J. Van Tilborg, K. Nakamura, C. G. R. Geddes, C. B. Schroeder, E. Esarey, and W. P. Leemans, "Control of tunable, monoenergetic laser-plasma-accelerated electron beams using a shock-induced density downramp injector," Phys. Rev. Accel. Beams 20, 051301 (2017).

${ }^{39}$ J. Xu, A. Buck, S.-W. Chou, K. Schmid, B. Shen, T. Tajima, M. C. Kaluza, and L. Veisz, "Dynamics of electron injection in a laser-wakefield accelerator," Phys. Plasmas 24, 083106 (2017).
${ }^{40}$ H. Ekerfelt, M. Hansson, I. Gallardo González, X. Davoine, and O. Lundh, “A tunable electron beam source using trapping of electrons in a density down-ramp in laser wakefield acceleration," Sci. Rep. 7, 12229 (2017).

${ }^{41}$ F. Massimo, A. F. Lifschitz, C. Thaury, and V. Malka, "Numerical studies of density transition injection in laser wakefield acceleration," Plasma Phys. Controlled Fusion 59, 085004 (2017).

${ }^{42}$ A. Martinez De La Ossa, Z. Hu, M. J. Streeter, T. J. Mehrling, O. Kononenko, B. Sheeran, and J. Osterhoff, "Optimizing density down-ramp injection for beam-driven plasma wakefield accelerators," Phys. Rev. Accel. Beams 20, 091301 (2017).

${ }^{43}$ H.-E. Tsai, K. K. Swanson, S. K. Barber, R. Lehe, H.-S. Mao, D. E. Mittelberger, S. Steinke, K. Nakamura, J. Van Tilborg, C. Schroeder, E. Esarey, C. G. R. Geddes, and W. Leemans, "Control of quasi-monoenergetic electron beams from laser-plasma accelerators with adjustable shock density profile," Phys. Plasmas 25, 043107 (2018).

${ }^{44}$ K. N. Kim, Y. Hwangbo, S.-g. Jeon, and J. Kim, "Characteristics of the shock structure for transition injection in laser wakefield acceleration," J. Korean Phys. Soc. 73, 561 (2018).

${ }^{45}$ S. K. Barber, J. Van Tilborg, C. B. Schroeder, R. Lehe, H.-E. Tsai, K. K. Swanson, S. Steinke, K. Nakamura, C. G. R. Geddes, C. Benedetti, E. Esarey, and W. P. Leemans, "Parametric emittance measurements of electron beams produced by a laser plasma accelerator," Plasma Phys. Controlled Fusion 60, 054015 (2018).

${ }^{46}$ A. Adelmann, B. Hermann, R. Ischebeck, M. Kaluza, U. Locans, N. Sauerwein, and R. Tarkeshian, "Real-time tomography of gas-jets with a Wollaston interferometer," Appl. Sci. 8, 443 (2018).

${ }^{47}$ Y. Wu, L. Ji, X. Geng, Q. Yu, N. Wang, B. Feng, Z. Guo, W. Wang, C. Qin, X. Yan, L. Zhang, J. Thomas, A. Hützen, A. Pukhov, M. Büscher, B. Shen, and R. Li, "Polarized electron acceleration in beam-driven plasma wakefield based on density down-ramp injection,” Phys. Rev. E 100, 043202 (2019).

${ }^{48}$ M. Fang, Z. Zhang, W. Wang, J. Liu, and R. Li, “Sharp plasma pinnacle structure based on shockwave for an improved laser wakefield accelerator," Plasma Phys. Controlled Fusion 60, 075008 (2018).

${ }^{49}$ A. M. Hansen, D. Haberberger, J. Katz, D. Mastrosimone, R. K. Follett, and D. H. Froula, "Supersonic gas-jet characterization with interferometry and Thomson scattering on the OMEGA laser system," Rev. Sci. Instrum. 89, 10C103 (2018).

${ }^{50} \mathrm{~J}$. Gomez and R. Groll, "Pressure drop and thrust predictions for transonic micronozzle flows," Phys. Fluids 28, 022008 (2016).

${ }^{51}$ S. Lorenz, G. Grittani, E. Chacon-Golcher, C. M. Lazzarini, J. Limpouch, F. Nawaz, M. Nevrkla, L. Vilanova, and T. Levato, "Characterization of supersonic and subsonic gas targets for laser wakefield electron acceleration experiments," Matter Radiat. Extremes 4, 015401 (2019).

${ }^{52}$ R. Octavianty and A. Bramhasta, in Proceedings of the 3rd International Seminar on Metallurgy and Materials (ISMM2019): Exploring New Innovation in Metallurgy and Materials (AIP Publishing, 2020), Vol. 2232, p. 020007.

${ }^{53}$ A. H. Epstein, MIT Gas Turbine Lab Report 117, 1974.

${ }^{54}$ R. K. Hanson and J. M. Seitzman, in Handbook of Flow Visualization (Routledge, 2018), pp. 225-237.

${ }^{55} \mathrm{H}$. M. Mott-Smith, "The solution of the Boltzmann equation for a shock wave," Phys. Rev. 82, 885 (1951).

${ }^{56}$ G. R. Plateau, N. H. Matlis, C. G. R. Geddes, A. J. Gonsalves, S. Shiraishi, C. Lin, R. A. Van Mourik, and W. P. Leemans, "Wavefront-sensor-based electron density measurements for laser-plasma accelerators," Rev. Sci. Instrum. 81, 033108 (2010).

${ }^{57}$ T. C. Adamson and J. A. Nicholls, "On the structure of jets from highly underexpanded nozzles into still air,” J. Aerosp. Sci. 26, 16 (1959).

${ }^{58}$ C. J. Greenshields, H. G. Weller, L. Gasparini, and J. M. Reese, "Implementation of semi-discrete, non-staggered central schemes in a colocated, polyhedral, finite volume framework, for high-speed viscous flows," Int. J. Numer. Methods Fluids 63, 1 (2010).

${ }^{59}$ L. Fan-Chiang, H.-S. Mao, and W. P. Leemans, in 2018 IEEE Advanced Accelerator Concepts Workshop (AAC) (IEEE, 2018), pp. 1-4.

${ }^{60} \mathrm{~J}$. Anderson, Modern Compressible Flow: With Historical Perspective (McGrawHill, Boston, MA, 2003), pp. 377-430.

${ }^{61}$ C. N. Kleber and S. Jarvis, Frankfort Aresnal Report R-1107, 1952. 